\title{
Bayesian Monte Carlo assimilation for the PETALE experimental programme using inter-dosimeter correlation
}

\author{
Axel Laureau ${ }^{1, *}$, Vincent Lamirand ${ }^{1,2}$, Dimitri Rochman ${ }^{2}$, and Andreas Pautz ${ }^{1,3}$ \\ ${ }^{1}$ Laboratory for Reactor Physics and Systems behaviour (LRS), Ecole Polytechnique Fédérale de Lausanne (EPFL), CH-1015 Lau- \\ sanne, Switzerland \\ ${ }^{2}$ Laboratory for Reactor Physics and Thermal Hydraulics (LRT), Paul Scherrer Institut (PSI), CH-5232 Villigen, Switzerland \\ ${ }^{3}$ Nuclear Energy and Safety Research Division (NES), Paul Scherrer Institut (PSI), CH-5232 Villigen, Switzerland
}

\begin{abstract}
This article presents the methodology developed to generate and use dosimeter covariances and to estimate nuisance parameters for the PETALE experimental programme. In anticipation of the final experimental results, this work investigates the consideration of these experimental correlations in the Bayesian assimilation process on nuclear data. Results show that the assimilation of a given set of dosimeters provides a strong constraint on some of the posterior reaction rate predictions of the other dosimeters. It confirms that, regarding the assimilation process, the different sets of dosimeters are correlated.
\end{abstract}

\section{Introduction}

The PETALE experimental programme in the CROCUS reactor at EPFL intends to contribute to the improvement of neutron nuclear data in the $\mathrm{MeV}$ energy range for stainless steel, particularly in the prospect of heavy reflector elements of PWRs. It mainly consists in several transmission experiments with metal plates interleaved with thin dosimeter foils. These metal plates are composed of nuclear-grade stainless steel, and its elemental components - iron, nickel and chromium - separately to avoid compensation effects. After irradiation, the dosimeter activities are measured in High Purity Germanium (HPGe) gamma spectrometers to be compared with calculation results. In this frame, the experiments were first prepared and optimized using Total Monte Carlo (TMC) uncertainty propagation in association with a Correlated Sampling (CS) technique using a modified version of the Serpent2 Monte Carlo code [1]. This first step highlighted the high correlation level between the dosimeters regarding the effect of the nuclear data uncertainties.

This article presents the Bayesian Monte Carlo (BMC) assimilation technique and the specific requirements of the PETALE programme in sec. 2. The methodology developed to generate dosimeter covariances from the experiment and the analysis is detailed in sec. 3 . In anticipation of the final experimental results, this work investigates in sec. 4 how these experimental correlations impact the Bayesian assimilation process on nuclear data.

\section{BMC for integral experiments}

\subsection{Principle}

Bayesian inference consists in assimilating a new information in a prior knowledge. Applied to nuclear data, the $\mathrm{BMC}$ approach uses a Monte Carlo procedure on the prior knowledge - random cross sections - and a weight is associated to each sample according to the agreement with the experiment:

$$
w_{x}=\exp \left(-\frac{\chi^{2}}{2}\right)
$$

Associated to the TENDL-2017 [2] library that applies the cross section generation from the nuclear parameters directly, the BMC assimilation consists in the TMC [3] uncertainty propagation procedure to obtain the $\chi^{2}$ plus the weight calculation using eq. 1 . No first order approximation is required, and any calculated observable can be used as long as a $\chi^{2}$ can be calculated.

The principal drawback is the important computation time due to the different required calculations with the sampled set of cross sections. In addition, if the calculations are done using a Monte Carlo code, then the statistical uncertainties have to be smaller that the experimental uncertainty to avoid a numerical bottleneck.

\subsection{PETALE programme and specific assimilation requirements}

The PETALE setup is a transmission experiment trough eight metal plates of $2 \mathrm{~cm} \times 32 \mathrm{~cm} \times 32 \mathrm{~cm}$ interleaved with thin dosimeter foils as represented in fig. 1. Thus the neutron transmission is assessed using neutron activation analysis: depending on the dosimeter material chosen, the

*e-mail: axel.laureau@epfl.ch 
measurement will be representative of different parts of the spectrum. The complete experiment description is available in [4].
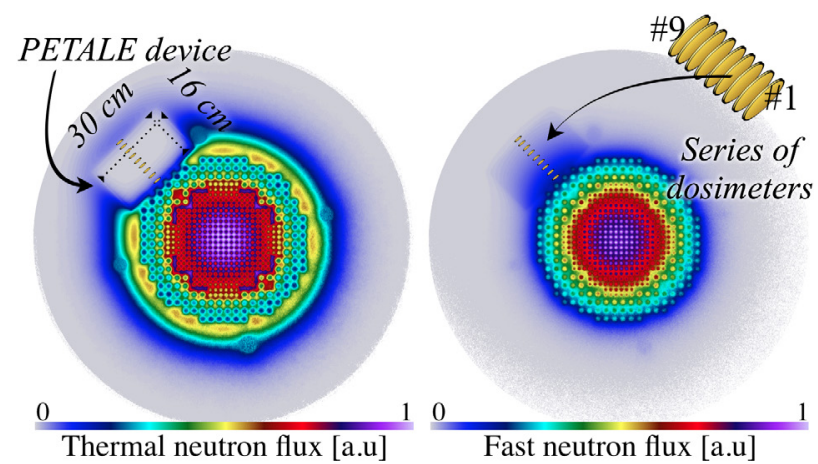

Figure 1. Thermal (left) and fast (right) neutron flux in the CROCUS reactor with the iron setup of the PETALE programme calculated with the Serpent 2 code.

Associating results of different dosimeters allows to decrease the experimental uncertainties. For example, using the ratio of two dosimeter activities, the uncertainty on the dosimeter cross section should be suppressed since the relative impact on the reaction rate is almost the same. When using two dosimeter materials at the same position, the reactor power uncertainty is suppressed. The later is similar to a classic spectral index with fission chambers but with dosimeters.

The objective of this work is to perform the assimilation on all the dosimeters in a generic way. For this, all the dosimeters have to be considered together. Then a generic covariance of the experiment containing all the dosimeters is required.

Note that in this article, we focus on the dosimeter analysis of the iron phase of the PETALE programme as an example for illustration purpose.

\section{Measurement correlation}

\subsection{Overall principle}

In order to obtain the calculation-experiment $\chi^{2}$, one needs to link the raw measured counts in the HPGe and the random cross sections from the TENDL library. To do so, we need to link both sides up to the reaction rate in the dosimeters:

- Thanks to the HPGe calibration, we obtain a dosimeter activity from measured counts. Then solving the Bateman equation with the power history and the power calibration, we get the reaction rate per Joule in the reactor.

- Using a Monte Carlo calculation for each random cross section file, a value of reaction rate per Joule is also obtained.

\subsection{Uncorrelated contributors}

In the calculation-experiment comparison process, some of the uncertainties are not correlated between the dosimeters.

The number of counts in the HPGe is not correlated between the dosimeters. The standard deviation is directly given by the square root of the number of counts and the covariance is zero. In this study, we consider the following achievable order of magnitude: $1 \%$ uncertainty for the dosimeters sensitive to thermal neutrons, and $2 \%$ for the threshold dosimeters.

The self-absorption of the $\gamma$-rays in the dosimeters and the geometrical correction (plane dosimeter vs pointlike calibration source of ${ }^{152} \mathrm{Eu}$ ) are estimated with Monte Carlo calculation and, since all the dosimeters are different, the statistical uncertainty is not correlated. Moreover, this uncertainty can be reduced to a value much smaller than $0.1 \%$.

The last uncorrelated uncertainty is the statistical uncertainty of the neutron transport Monte Carlo. This uncertainty is linked to the number of propagated particles in the dosimeters. The order of magnitude is around $1 \%$ and can be decreased using a longer calculation.

\subsection{HPGe efficiency}

A good HPGe efficiency calibration is important to estimate the dosimeter activity from the measured number of counts. This calibration has an uncertainty due to the fitting procedure detailed below, and from the calibration source (Europium here) activity that has an uncertainty $(0.5 \%$ at $1 \sigma$ in this study).

The uncertainty of the calibration source activity is a systematic uncertainty, meaning that it is fully correlated between the dosimeters and that comparing ratios of activities suppresses this uncertainty. The uncertainty on the fitting procedure is not a systematic uncertainty when comparing different energies and has to be estimated precisely.

Considering the fitting of the efficiency shape, we consider a ${ }^{152} \mathrm{Eu}$ source providing different fitted efficiency points represented in fig. 2 with their statistical uncertainties.
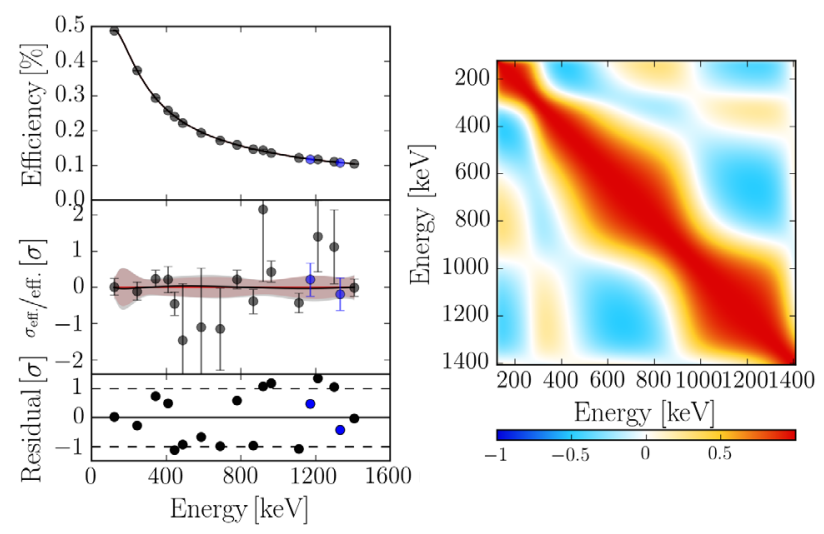

Figure 2. Left part: HPGe efficiency (top) calculated using ${ }^{152} \mathrm{Eu}$ source (points and error bars) and fit (plain line), zoom on the relative uncertainty (middle) and residuals (bottom). Right part: correlation matrix associated to the fit represented on the left part.

Using different dosimeter materials, the $\gamma$-ray emissions are at different energies. For example, ${ }^{115} \operatorname{In}(n, n ')$ reaction is emitting a $\gamma$-ray at $336 \mathrm{keV}$ and ${ }^{59} \mathrm{Co}(\mathrm{n}, \gamma)$ at 1332 $\mathrm{keV}$. For those two gamma rays, from fig. 2 the correlation between the dosimeters appears to be small, meaning that the impact of the efficiency uncertainty is maximal. On the 
other hand, beetween ${ }^{115} \operatorname{In}(\mathrm{n}, \gamma)$ reaction at $1293 \mathrm{keV}$ and ${ }^{59} \mathrm{Co}$ at $1332 \mathrm{keV}$, this point is very close to the diagonal of the correlation matrix, and therefore, the impact of the efficiency uncertainty is almost zero here.

\subsection{Dosimeter cross sections}

In order to estimate the reaction rate from the Monte Carlo calculation, dosimeter cross sections are required. These cross sections have an associated uncertainty and correlation matrix that have to be propagated. In this study we consider the IRDFF-1.05 nuclear data dosimetry library [5] as represented in fig. 3 for gold dosimeters.
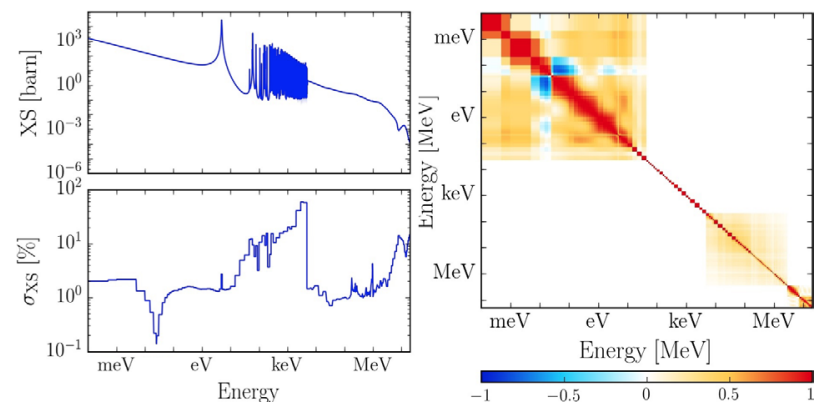

Figure 3. Gold capture cross section (top-left) together with the associated uncertainty (bottom-left) and correlation matrix (right) from the IRDFF-1.05 nuclear data library [5]

The neutron flux is estimated by Monte Carlo calculation in all the dosimeters located between each metal plate. In order to estimate the correlation between the different dosimeters, the reaction rate spectrum (i.e. energy contribution to the reaction rate) is also scored using the Monte Carlo calculation. We can see on the seconde graph of fig. 4 that the reaction rate is mainly concentrated at the gold resonance energy, except for the first (blue) and last (red) curves that correspond to the first and last dosimeters with a more important thermal component.
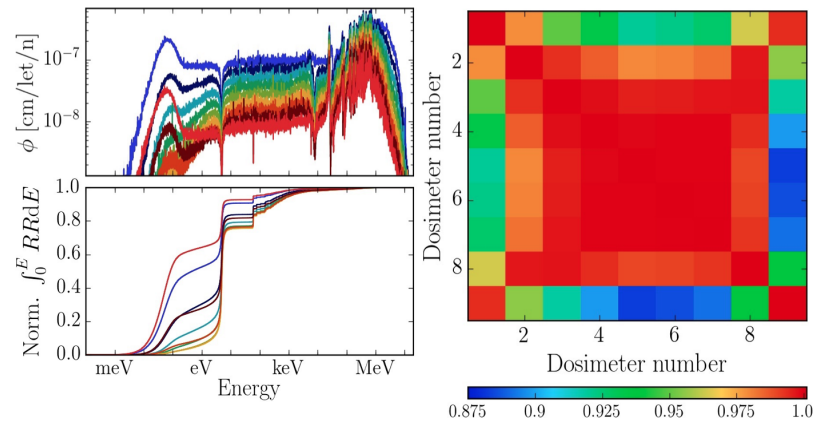

Figure 4. Neutron flux (top-left) in the dosimeter from the closest (blue) to the farthest (red) away from the core, and the corresponding reaction rate distribution (bottom-left) integrated and normalized to one to see the difference in the contribution. The dosimeter correlation matrix (right) is computed using the IRDFF covariance matrix on the cross sections.

Note that, for the dosimeters located deep inside the PETALE setup, the correlation is close to $100 \%$ (in red in fig. 4 - right). It is important to take into account that the correlation is lower (in blue and green) for the dosimeters near to the border of the heavy metal reflector and then the corresponding uncertainty cannot be completely suppressed using a ratio of reaction rates. For this reason this matrix has to be estimated for all the dosimeter materials.

\subsection{Material and geometry nuisance parameters}

The material and geometry nuisance parameters are estimated using a Monte Carlo approach. The uncertainty on the material cross sections is estimated using multiple calculations associated to the TENDL library with the correlated sampling technique as for the iron itself [1]. For example, in the case of the aluminium box around the metal plates, the uncertainty is below $0.5 \%$.

The propagation of the geometrical uncertainties is done with a random sampling of all the geometric parameters (width, distance...) with an amplification of the uncertainty range in order by a factor 5 in order to help the estimation of the reaction rate variation between independent Serpent 2 calculations as illustrated in fig. 5. The geometrical uncertainty is around $1 \%$ divided by 5 , and the correlation between the dosimeters tends to decrease the impact of this uncertainty when comparing reaction rates.

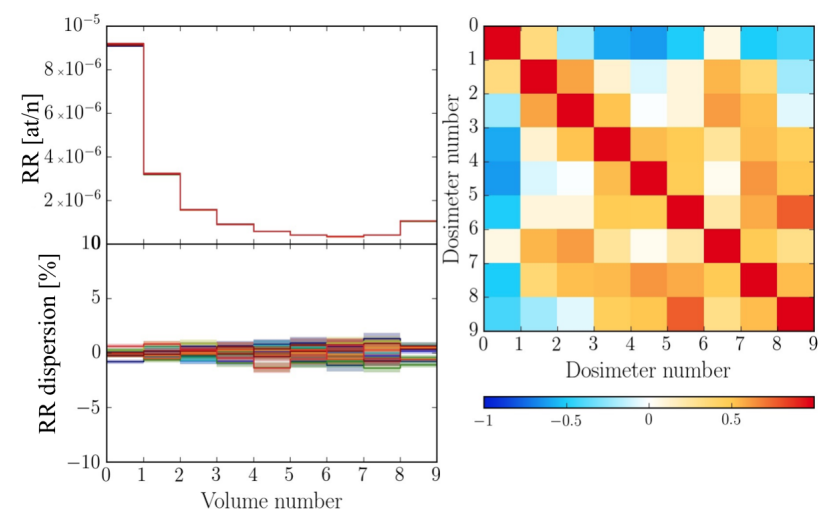

Figure 5. Reaction rate of indium dosimeter capture reaction for the different dosimeters (top left) and the associated relative variation when perturbating the geometry (bottom left) and the associated correlation matrix (right).

\section{Assimilation with covariances}

\subsection{Propagation in the BMC technique}

The BMC requires a $\chi^{2}$ value associated to each calculated value (i.e. TENDL file). To compute this $\chi^{2}$, we use the calculated value from the Serpent 2 calculation for each iron TENDL file (all isotopes included). An experimental value is also required. Since the experiment is not done yet, we consider in this preliminary study a best case scenario with an experimental value equal to the average of the calculated values. For the correlation values, we use the matrices discussed previously computed for all the dosimeter materials and positions.

\subsection{Assimilation of the dosimeter reaction rates}

The prior uncertainty distribution is presented in fig. 6 using 256 random iron cross sections. We observe that the 
dosimeters on the right of the figure have the smallest reaction rate and the highest propagated uncertainty (up to $15 \%)$. Indeed the ordering of the dosimeters is done using the average neutron energy associated to the reaction on the dosimeters: from $\sim 1.7 \mathrm{eV}$ for indium $(\mathrm{n}, \gamma)$ to $\sim 10 \mathrm{MeV}$ for vanadium.

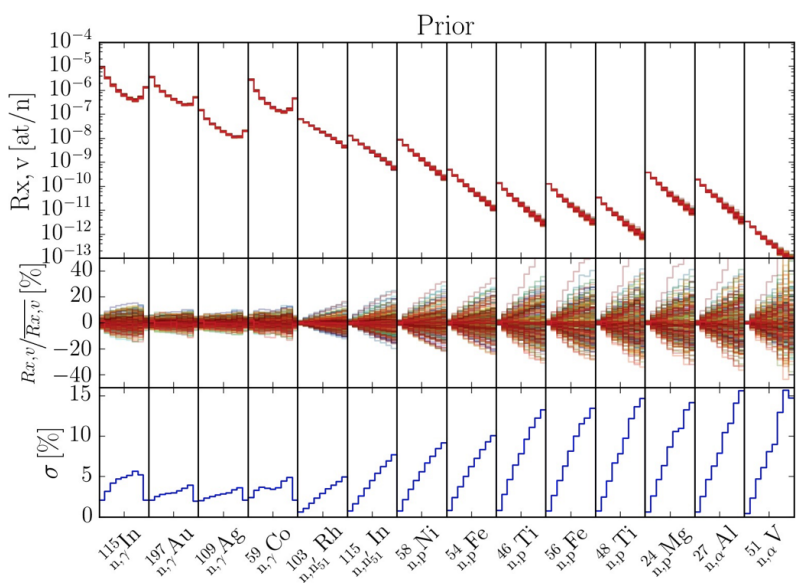

Figure 6. Prior dosimeter reaction rates (top) with the different dosimeter numbers time materials as abscissa. The middle part represents the reaction rate prior dispersion due to the nuclear data uncertainty on iron (all isotopes) and the bottom represents the standard deviation.

Then performing an assimilation based for example on the nine dosimeters (different position) of cobalt and indium $(n, n ')$ reaction, the posterior distributions are represented in fig. 7.

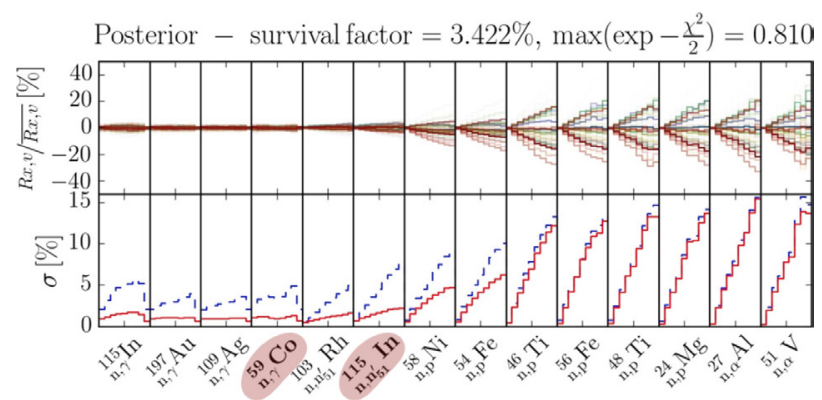

Figure 7. Posterior reaction rate distribution (top) and standard deviation (bottom) using the same abscissa as fig. 6 for an assimilation of ${ }^{59} \mathrm{Co}(\mathrm{n}, \gamma)$ and ${ }^{115} \mathrm{In}\left(\mathrm{n}, \mathrm{n}^{\prime}\right)$ dosimeters.

We observe that the dosimeter dispersion is reduced, as expected, for the two considered reactions. Note that the propagated uncertainties on all the other neutron capture reactions $\left({ }^{115} \mathrm{In},{ }^{197} \mathrm{Au},{ }^{109} \mathrm{Ag}\right)$ and for the inelastic ${ }^{103} \mathrm{Rh}$ and $(\mathrm{n}, \mathrm{p})$ reaction on ${ }^{58} \mathrm{Ni}$ and ${ }^{54} \mathrm{Fe}$ dosimeter are also decreased. This means that the dosimeters are not independent regarding the cross section uncertainty on which we perform the assimilation. This is consistent with the correlation matrix obtained using the prior uncertainty (middle part of fig. 6) represented in fig. 8: the first four dosimeters are fully correlated and the indium is correlated with rhodium, and slightly correlated with nickel and iron.

This confirms the choice of using at least one dosimeter of each sub-matrix of fig. 8 for the experimental programme [7]. The same BMC assimilation will be applied

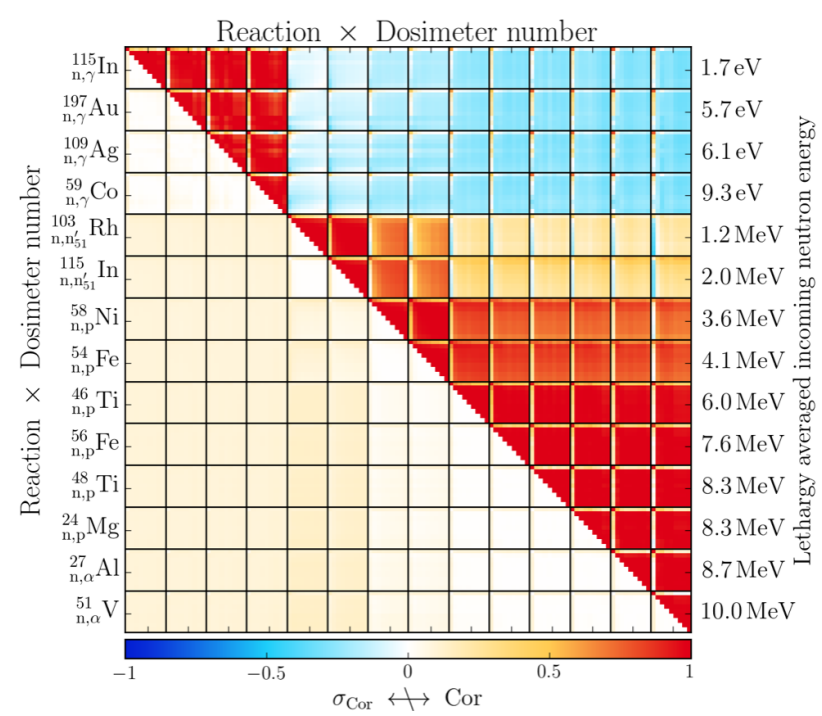

Figure 8. Prior reaction rate correlation due to iron cross section uncertainty with its standard deviation estimated with a Jackknife resampling technique [6].

to these dosimeters and the real data. Depending on the difference between the prior and the measured reaction rates, the number of required random cross sections can be much larger to obtain a converged posterior dispersion.

\section{References}

[1] A. Laureau, V. Lamirand, D. Rochman, A. Pautz, Total Monte Carlo acceleration for the PETALE experimental programme in the CROCUS reactor, in EPJ Web of Conferences (EDP Sciences, 2019), Vol. 211, p. 03002

[2] D. Rochman, A.J. Koning, J.C. Sublet, M. Fleming, E. Bauge, S. Hilaire, P. Romain, B. Morillon, H. Duarte, S. Goriely et al., The TENDL library: Hope, reality and future, in EPJ web of conferences (EDP Sciences, 2017), Vol. 146, p. 02006

[3] D. Rochman, A. Koning, S. van der Marck, A. Hogenbirk, D. van Veen, Journal of Korean Physics 59, 1236 (2011)

[4] V.P. Lamirand, G. Perret, S. Radman, D.J. Siefman, M. Hursin, P. Frajtag, A. Gruel, P. Leconte, P. Blaise, A. Pautz, Design of Separated Element Reflector Experiments in CROCUS: PETALE, in ISRD16 International Symposium on Reactor Dosimetry (2017), CONF

[5] E.M. Zsolnay, N. Capote, H.J. Nolthenius, A. Trkov et al., Tech. rep., International Atomic Energy Agency (2012)

[6] B. Efron, Biometrika 68, 589 (1981)

[7] V. Lamirand, A. Laureau, D. Rochman, G. Perret, A. Gruel, P. Leconte, P. Blaise, A. Pautz, An Experimental Programme optimized with Uncertainty Propagation: PETALE in the CROCUS Reactor, in EPJ Web of Conferences (EDP Sciences, 2019), Vol. 211, p. 03003 